American Journal of Infectious Diseases 7 (4): 104-106, 2011

ISSN 1553-6203

(C) 2011 Science Publications

\title{
Drug Susceptibility Pattern of M.Tuberculosis Isolated from Patients Attending a Private Hospital
}

\author{
Sureshkumar, D. and Ram Gopalakrishnan \\ Old No 128, New No 2, Raghavan Street, \\ Perambur, Chennai, Tamilnadu, 600 011, India
}

\begin{abstract}
Problem statement: Drug resistance in Tuberculosis (TB) is an emerging problem that adversely affects patient outcome and public health in the developing world. Although much of tuberculosis care is provided by the private sector in India, the magnitude of drug resistance in TB in the private sector is not well described. The present study was carried out to determine the resistance pattern of tuberculosis in patients attending a large tertiary care hospital in South India. Approach: Anti-tuberculosis resistance patterns of all Mycobacterium tuberculosis (M.tb) isolates in a tertiary care referral hospital from January 2010 to December 2010 were studied retrospectively. Isolates were grown in MB/BacT automated liquid culture system. Sensitivities to various anti-tuberculosis drugs were done by the proportion method on Lowenstein-Jensen (L-J) media. Results: During the study period, sensitivity reports for 50 Mycobacterium isolates were available.14 (28\%) of the isolates were multi-drug resistant isolates (resistant to both isoniazid and rifampicin). Isolated resistance to isoniazid, rifampicin, ethambutol, pyrazinamide and streptomycin were 42, 32, 28, 48.28 and $44 \%$ respectively. Moxifloxacin was tested against only 12 tuberculosis isolates and was uniformly sensitive against all isolates tested. Conclusion: Nearly one third of $M . t b$ isolates in a private sector tertiary care hospital were multi-drug resistant. Isolated resistance to ethambutol was the lowest among the first line antituberculosis drugs and resistance to moxifloxacin was not seen in this study. Even allowing for referral bias, our results suggest that tertiary care hospitals which see complicated tuberculosis patients should routinely ask for susceptibility tests whenever M.tb is cultured.
\end{abstract}

Key words: Tuberculosis, Drug resistance, Multidrug-resistance, Private health care

\section{INTRODUCTION}

Drug resistant Tuberculosis (TB) is a not a new phenomenon and has been known from the time when drugs were introduced for the treatment of TB. Drug resistant TB adversely affects patient outcome and public health in the developing world. The past two decades have seen the global appearance of MultidrugResistant Tuberculosis (MDR-TB) (Frieden et al., 1993; Robert et al., 2003), followed by Extensively Drug-Resistant Tuberculosis (XDR-TB) (Shah et al., 2007) and of late strains those are resistant to all antituberculosis drugs (Velyati et al., 2009). Data from the published literature are insufficient to indicate whether the incidence of Multidrug-Resistant (MDR) TB is rising or falling globally as many national programs are failing to diagnose MDR tuberculosis. Globally, only 30, 000 cases of MDR-TB were reported to World Health Organization (WHO) in 2008, compared to the estimate of 440,000 cases (7\% of the estimated total). The WHO/IUALTD Global project on Anti-tuberculosis Drug Resistance Surveillance recorded considerable variation in the prevalence of drug resistance among 35 countries in 5 different continents (Velyati et al., 2009).

Drug susceptibility testing in India is not routinely performed and public health laboratory infrastructure is limited and poorly equipped to cope with large scale testing. Most of the published reports on tuberculosis drug resistance in India came from surveillance studies conducted in outpatient settings under Revised National TB Control Program (RNTCP) (Paramasivan et al., 2002; 2000). However a substantial proportion of patients with tuberculosis or drug resistant tuberculosis seek care with private care providers International Institute of Population Sciences IIPS, 2007. In countries with high burden of MDR tuberculosis, more than half of all sales of first line antituberculosis drugs occur in the private care sector and the proportion is even higher for sales of second-line

Corresponding Author: Sureshkumar, D., Old No 128, New No 2, Raghavan Street, Perambur, Chennai, Tamilnadu, 600 011, India Tel: 91944186807 
Am. J. Infect. Dis., 7 (4): 104-106, 2011

drugs (GATBDD, 2007). The information on anti tuberculosis drug resistance from private health care sector is localized within certain regions of India (Rodrigues et al., 2006). The aim of the present study was to describe the pattern of drug resistance among $M . t b$ isolates in patients attending a private tertiary care referral hospital in South India.

\section{MATERIALS AND METHODS}

This was a retrospective, descriptive, laboratory based study of tuberculosis isolates from the patients attending a 600 bed tertiary care hospital located in Chennai, South India between January 2010 and December 2010.

Isolates were grown in $\mathrm{MB} / \mathrm{BacT}$ automated liquid culture system. Sensitivities to various anti-tuberculosis drugs were done by the proportion method on Lowenstein-Jensen (L-J) media.

The following definition of resistance was followed in defining the isolates:

- Multidrug-resistance: resistance to both isoniazid $(\mathrm{H})$ and rifampicin $(\mathrm{R})$

- Monoresistance: resistance to only one drug

- Polyresistance: resistance to two or more drugs excluding HR resistance

- Drug sensitive: absence of resistance to any of the drugs

The ethical committee of the hospital gave ethical clearance for this study.

\section{RESULTS}

During the study period, drug susceptibility results were available for 50 of the M.tb isolates. Of the 50 isolates $14(28 \%)$ were drug sensitive isolates (susceptible to all the anti-tuberculosis drugs) and 14 were MDR isolates. Poly-resistant isolates were the predominate isolates $(15 / 50-30 \%)$ in this study and mono-resistant isolates were seen only in 7 isolates $(14 \%)$, but none of the isolates were extremely drug resistant (XDR) TB isolates in this study.

As shown in Table 1 among the individual first line drugs the resistance rates were $48.28,44,42,32$ and $28 \%$ for pyrazinamide, streptomycin, isoniazid, rifampicin and ethambutol respectively. For the second line drugs resistance rate were $32.26 \%$ for ethionamide and $17.22 \%$ for amilkacin. Moxifloxacin was the only drug sensitive $100 \%$ of the time (13/13 of the isolates) in this study.
Table 1: Drug susceptibility patterns of Mycobacterium tuberculosis isolates to first and second line drugs

\begin{tabular}{llcr}
\hline $\begin{array}{l}\text { Drugs } \\
\text { (Total no isolates tested) }\end{array}$ & $\begin{array}{l}\text { Sensitive } \\
(\mathrm{N})\end{array}$ & $\begin{array}{c}\text { Resistant } \\
(\mathrm{N})\end{array}$ & $\begin{array}{l}\text { Resistance rate } \\
(\%)\end{array}$ \\
\hline Isoniazid (50) & 29 & 21 & 42.00 \\
Rifampicin (50) & 34 & 16 & 32.00 \\
Pyrazinamide (29) & 15 & 14 & 48.28 \\
Ethambutol (50) & 36 & 14 & 28.00 \\
Stretomycin(50) & 28 & 22 & 44.00 \\
Amikacin (29) & 24 & 5 & 17.22 \\
Etionamide (37) & 21 & 16 & 32.26 \\
Moxifloxacin (12) & 12 & 0 & 0.00 \\
\hline
\end{tabular}

\section{DISCUSSION}

Our study found a high prevalence of drug resistance to at least one anti-tuberculosis drug (72\%) and an MDR-TB prevalence of $28 \%$ in patient attending our tertiary care hospital in South India. The estimated prevalence of MDR-TB in our study was less than in other study conducted in a tertiary hospital in Mumbai (Almedia et al., 2003) (resistance to any one drug 80\% and MDR-TB 51\%). However it was substantially higher than rates found in a number of surveillance studies conducted in RNTCP programs (resistance to any one drug was $21-46 \%$ and MDR-TB 2.4-17.4\%) (Chandrasekaran et al., 1990; Anuradha et al., 2006; Ramachandran et al., 2009). Bias in the selection and differences in the methodology may explain such variations in the prevalence rates.

The resistance rates noticed in our study to isoniazid and rifampicin were 42 and $32 \%$ respectively. Previous surveillance studies conducted in the state of Gujarat (Ramachandran et al., 2009) showed that the frequency of INH and rifampicin resistance were in the range of $11-37 \%$ for INH and $2.5 \%-18 \%$ for rifampicin and the study conducted by the Deodhar et al. (1999) in Bombay showed similar rates to our study (H-30.41\%, R- 58.55\%). This high rate of INH and rifampicin resistance may be due to wide spread use of these drugs in first line treatment of patients and possibly irregular and inadequate dosing of the drug. In our study, the highest (48.28\%) resistance among the first line drugs was found against pyrazinamide. PZA susceptibility testing is not routinely recommended as it acts at a lower $\mathrm{pH}$ where bacteria do not grow, but for special reasons it can be performed in small number of isolates (UNION/WHO, 1994; Mitchison, 2005). In our study ethambutol had the lowest resistance rate among the first line anti-TB drugs (28\%), similar to previous surveillance studies (1.9-10.2\%). This advantage should be exploited while constructing an effective empiric second line regimen for MDR-TB.

Among the second line drugs, ethionamide has got maximum resistance in our study with $32.26 \%$, a 
prevalence rate similar to studies conducted in the RNTCP (25-40.5\%) (Ramachandran et al., 2009). Resistance to ethionamide is difficult to interpret because of technical reasons (Mitchison, 2005). In addition there may be cross-resistance with INH due to a mutation in the inhA gene. Moxifloxacin is the only drug that retained $100 \%$ susceptibility in this study; no other studies in India tested this drug. This $100 \%$ susceptibility seen in our study could be due to very limited use of this drug in both the private sector and in national programs due to its high cost. Resistance to streptomycin was much higher when compared to amikacin in this study (44 Vs $17.22 \%$ ). Streptomycin resistance is a concern as it is an essential component of category II DOTS regimen.

Our study has limitations. Clearly there may be a referral bias towards sicker patients and relapses of TB in a referral hospital. Patients' clinical data, drug exposure history and outcomes were not analyzed.

\section{CONCLUSION}

In conclusion we found that up to $28 \%$ of patients in private settings may have MDR-TB. We recommend routinely doing mycobacterial cultures on clinical samples from all patients with TB and drug-susceptibility testing on all isolates of M.tb. There is clearly a need to further study anti-TB drugs resistance in hospital based TB patients from private health care institutions.

\section{REFERENCES}

Almedia, D., C. Rodrigues, Z.F. Udwadia, A. Lalvani and G.D. Gothi et al., 2003. Incidence of multidrug-resistant tuberculosis in urban and rural India and implications for prevention. Clin. Infect. Dis., 36: e152-e154. DOI: 10.1086/374931

Anuradha, B., S. Aparna, V.H.S. Priya, V.V. Lakshmi and Y. Akbar et al., 2006. Prevalence of drug resistance under the DOTS strategy in Hyderabad, South India, 2001-2003. Int. J. Tuberc. Lung. Dis., 10: 58-62. PMID: 16466038

Chandrasekaran, S., M.M. Chauhan, R. Rajalakshmi, K. Chaudhuri and B. Mahadev, 1990. Initial drug resistance to anti-tuberculosis drugs in patients attending an urban district tuberculosis centre. Ind. J. Tub., 37: 215-216.

Deodhar, L., P. Miskeen and S. Chomal, 1999. Drug Resistance in tuberculosis. Department of Microbiology.
Frieden, T.R., T. Sterling, A. Pablos-Mendez, J.O. Kilburn and G.M. Cauthen et al., 1993. The emergence of drug-resistant tuberculosis in New York City. N Engl. J. Med., 328: 521-26. DOI: 10.1056/NEJM199302253280801

GATBDD, 2007. Pathway to patients: Charting the dynamics of the global TB drug market. Global Alliance for TB Drug Development.

Mitchison, D.A., 2005. Drug resistance in tuberculosis. Eur. Respir. J., 25: 376-379. DOI: 10.1183/09031936.05.00075704

Paramasivan, C.N., K. Bhaskaran, P. Venkatraman, V. Chandrasekaran and P.R. Narayanan, 2000. Surveillance of drug resistance in tuberculosis in the state of Tamil Nadu. Ind. J. Tub., 47: 27-33.

Paramasivan, C.N., P. Venkatraman, V. Chandrasekaran, S. Bhat and P.R. Narayanan, 2002. Surveillance of drug resistance in tuberculosis in two districts of South India. Int. J. Tuberc. Lung. Dis., 6: 479-484. PMID: 12068979

Ramachandran, R., S. Nalini, V. Chandrasekar, P.V. Dave and A.S. Sanghvi et al., 2009. Surveillance of drug-resistant tuberculosis in the state of Gujarat, India. Int. J. Tuberc. Lung. Dis., 13: 1154-1160. PMID: 19723407

Robert, J., D. Trystram, C. Truffot-Pernot and V. Jarlier, 2003. Multidrug-resistant tuberculosis: Eight years of surveillance in France. Eur. Respir. J., 22: 833-837. DOI: 10.1183/09031936.03.00014103

Rodrigues, C., S. Shenai, M. Sadani, P. Thakkar and A. Sodha et al., 2006. Multidrug-resistant tuberculosis in Mumbai: it's only getting worse. Int. J. Tuberc. Lung. Dis., 10: 1421-1422. PMID: 17171830

Shah, N.S., A. Wright, G.H. Bai, L. Barrera and F. Boulahbal et al., 2007. Worldwide emergence of extensively drug-resistant tuberculosis. Emerg. Infect. Dis., 13: 380-87. DOI: 10.3201/eid1303.061400

Velyati, A.A., M.R. Masjedi, P. Farina, P. Tabarsi and J. Ghanavi et al., 2009. Emergence of new forms of totally drug-resistant tuberculosis bacilli super extensively drug-resistant tuberculosis or totally drug-resistant strains in Iran. CHEST, 136: 420425. DOI: 10.1378/chest.08-2427

UNION/WHO, 1994. Guidelines for Surveillance of Drug Resistance in Tuberculosis. 1st Edn., WHO, Geneva, pp: 24. 